\section{RMD Open}

Rheumatic \&

Musculoskeletal Diseases

\title{
Therapeutic implications of the anergic/ postactivated status of B cells in systemic lupus erythematosus
}

Thomas Dörner (D), ${ }^{1}$ Franziska Szelinski, ${ }^{2}$ Andreia C Lino, ${ }^{3,4}$ Peter E Lipsky ${ }^{5}$

To cite: Dörner T, Szelinski F, Lino AC, et al. Therapeutic implications of the anergic/ postactivated status of $B$ cells in systemic lupus erythematosus. RMD Open 2020;6:e001258. doi:10.1136/rmdopen-2020001258

Received 2 April 2020 Revised 28 May 2020 Accepted 12 June 2020
Check for updates

(c) Author(s) (or their employer(s)) 2020. Re-use permitted under CC BY-NC. No commercial re-use. See rights and permissions. Published by BMJ.

${ }^{1}$ Rheumatology, Charité Hospital, Berlin, Germany

${ }^{2}$ Charité - Universitätsmedizin Berlin, Berlin, Germany ${ }^{3}$ Department of Rheumatology and Clinical Immunology, Charité University Hospital, Berlin,

Germany

${ }^{4}$ German Rheumatism Research Center Berlin (DRFZ), Berlin,

Germany

${ }^{5}$ RILITE Research Institute, Charlottesville, Virginia, USA

Correspondence to Thomas Dörner; thomas.doer ner@charite.de

\section{ABSTRACT}

Systemic lupus erythematosus (SLE) is characterised by numerous abnormalities in B lineage cells, including increased CD27++ plasmablasts/plasma cells, atypical CD27-lgD- B cells with increased CD95, spleen tyrosine kinase (Syk)++, CXCR5- and CXCR5+ subsets and anergic CD11c+Tbet+ age-associated B cells. Most findings, together with preclinical lupus models, support the concept of $B$ cell hyperactivity in SLE. However, it remains largely unknown whether these specific B cell subsets have pathogenic consequences and whether they provide relevant therapeutic targets. Recent findings indicate a global distortion of $B$ cell functional capability, in which the entire repertoire of naïve and memory $B$ cells in SLE exhibits an anergic or postactivated (APA) functional phenotype. The APA status of SLE B cells has some similarities to the functional derangement of lupus T cells. APA B cells are characterised by reduced global cytokine production, diminished B cell receptor (BCR) signalling with decreased Syk and Bruton's tyrosine kinase phosphorylation related to repeated in vivo BCR stimulation as well as

hyporesponsiveness to toll-like receptor 9 engagement, but intact CD40 signalling. This APA status was related to constitutive co-localisation of CD22 linked to phosphatase SHP-1 and increased overall protein phosphatase activities Notably, CD40 co-stimulation could revert this APA status and restore BCR signalling, downregulate protein tyrosine phosphatase transcription and promote B cell proliferation and differentiation. The APA status and their potential rescue by bystander help conveyed through CD40 stimulation not only provides insights into possible mechanisms of escape of autoreactive clones from negative selection but also into novel ways to target B cells therapeutically.

\section{INTRODUCTION}

Loss of central and peripheral self-tolerance and subsequent maintenance of autoimmune memory by $\mathrm{T}$ and $\mathrm{B}$ lineage cells and the resultant autoantibody production are important pathologic features of adaptive immunity in systemic lupus erythematosus (SLE). ${ }^{1}{ }^{2}$ Various murine models of $\mathrm{SLE}^{34}$ point towards the key finding of hyperactive $\mathrm{B}$ cells driven by autoreactive $T$ cells and lack of certain tolerance checkpoints as important in the immunopathogenesis of SLE. In patients with SLE, specific abnormalities of peripheral B cell subsets have been identified. . $^{5-13}$ 14-16

Key functions of $\mathrm{B}$ cells include recognition of antigen by the $\mathrm{B}$ cell receptor (BCR) and subsequent downstream signalling and cellular activation. In addition, B cell activation is modulated by numerous other receptors, including CD40 and the endosomal toll-like receptors (TLRs) (especially TLR7 and TLR9). Functionally, B cells can contribute to adaptive immunity by secreting cytokines, suppressing adaptive immune responses and, most prominently, by differentiating into antibody-secreting plasmablasts/plasma cells (PB/ $\mathrm{PC})$. It is important to recognise that under most circumstances, full activation of naïve $B$ cells requires engagement of the BCR and also second signals, such as ligand-ligand interaction or cytokines crucial for B cell fate. ${ }^{17-19}$ As such, BCR signalling is considered to play a necessary but not sufficient role in the development and maintenance of autoimmunity. Importantly, there is general consensus that one central contributor to B cell hyperactivity and autoimmunity is pathologically increased BCR signalling. ${ }^{20-22}$ Key findings of abnormal BCR signalling in autoimmunity are mainly derived from studies in mice, in which BCR hyperreactivity has been found to be a main driver of autoimmunity. ${ }^{23}$ Less is known about the contribution of individual phenotypically identified B cell populations to the development of autoimmunity and whether abnormalities in BCR signalling contribute to expansion or functional perturbations of these B cell subsets.

\section{ABNORMALITIES OF B LINEAGE DIFFERENTIATION IN SLE}

Several studies validated that increased $\mathrm{PB} / \mathrm{PC}$ induction is a feature of active $\mathrm{SLE}^{81524}$ including the demonstration that the PC gene expression profile correlated with disease activity. ${ }^{25}$ 
Subsequent studies dissecting subgroups of $\mathrm{PB} / \mathrm{PC}$ in peripheral blood of $\mathrm{SLE}^{26}$ characterised at least two subsets. One phenotype, expressing CCR10 and B7, produced IgA, whereas another subtype, expressing CD62L, produced preferentially IgG. Both contributed to the peripheral plasmocytosis, whereas mainly the latter were found in kidney infiltrates. The two subsets contained autoantibodyproducing cells with substantially different phenotypic and functional characteristics. It is not known whether their site of induction (mucosal immunity vs lymphoid organs) or germinal centre (GC) programming are different (GC vs extrafollicular).

The nomenclature and phenotypes of human B cells, such as transitional 1 and 2, resting and activated-naïve, preswitched and switched peripheral as well as tissueresident memory B cells, marginal zone B cells, regulatory B lineage cells, subsets of antibody-secreting cells (ASCs), ${ }^{27} 28$ have been described recently. Remaining challenges $^{28}$ lacking consensus have also been addressed, such as the nature of human $\mathrm{B} 1$ cells (CD27 +IgD++, IgM+, CD43+, CD70-, CD11c+, CD14+, CD5+-), regulatory $\mathrm{PC}(\mathrm{CD} 27++\mathrm{CD} 38++\mathrm{CD} 19 \pm$, interleukin (IL) $10+$ or IL-35+IgM++ or IgA+) and anergic-naïve B cells (IgD+, CD27-, CD38+/low, CD24+, CD21-, IgMlow/-). These are important references to evaluate abnormalities of peripheral and tissue-based B lineage cells in certain diseases, such as SLE. However, a main challenge is to understand the role of expanded atypical memory B cells in particular in SLE. Herein, we use the term atypical memory to refer to $\mathrm{B}$ cells that have been described or considered as antigen experienced, but do not have the classic phenotype of memory $\mathrm{B}$ cells.

The CD27-IgD- B cell population is the origin of many phenotypical studies of atypical B cell subsets in SLE. A better understanding of the role of the CD27-IgDpopulation and its subsets seems to be of special interest, since these cells are expanded in various autoimmune diseases, including SLE, ${ }^{10}$ rheumatoid arthritis (RA) and inflammatory bowel disease. ${ }^{29} 30$

In mice, a B cell subset termed age-/autoimmuneassociated $\mathrm{B}$ cells (ABCs) has been described that arises with age in normal mice and is expanded in various autoimmune diseases. ${ }^{31}$ A similar B cell subset has been reported in human autoimmune disease, although the similarity is based on limited phenotypic analysis. ABClike B cells in human autoimmunity are characterised by expression of CD19 ${ }^{\text {hi }}$ CD27- CD21- CD11c++ and the transcription factor Tbet+ (tbx21). Further markers and their expression levels have been reported, including CD23CD24 ${ }^{\text {lo }}$ CD38 ${ }^{\text {lo }}$ IL-4R ${ }^{\text {lo }}$ CD95 ${ }^{\text {hi }}$ CD86+ FCRL $5+.{ }^{16}$ Somatic hypermutation of BCR gene rearrangements, lack of expression of the ABCB1 transporter and the finding that the majority of the population is class switched mark them as memory B cells despite lacking CD27 expression. Reduced responsiveness to $\mathrm{BCR},{ }^{32} \mathrm{CD} 40^{33}$ or $\mathrm{TLR}^{32} 33$ stimulation suggested their exhausted phenotype is caused by persisting antigen-driven stimulation. Accordingly, the
ABC-like B cell population is expanded in autoimmune patients with RA, ${ }^{34}$ SLE, ${ }^{35}$ primary Sjögren's syndrome (pSS) ${ }^{36}$ and chronic infection, such as malaria. ${ }^{37}$ Enrichment of poly-reactive and autoreactive clones in ABC-B cells was taken as indicator of their pathogenic role. A functional study of $\mathrm{ABCs}^{38}$ in a B cell-intrinsic Ship-deficient $\left(\right.$ Ship $^{\Delta \mathrm{B}}$ ) lupus model provided further evidence that young mice already had increased CD11c+ ABC in spleen and lymph nodes. Later in life, those mice developed increased $\mathrm{T}$ cell activation linked to increased autoimmunity. Ship ${ }^{\Delta \mathrm{B}}$ follicular B cells showed the same potency in inducing $\mathrm{T}$ cell activation in an antigen-dependent manner as wild-type mice, whereas $\mathrm{CD} 11 \mathrm{c}+\mathrm{ABCs}$ were more potent stimulators of antigen-specific T cells with a TFH phenotype. Inducible deletion of CD11c resulted in a decrease of CXCR5+ PD- $1^{\text {hi }}$ $\mathrm{TFH}$, suggesting an important role of CD11c $+\mathrm{ABCs}$ for maintenance of TFH. Of note, antibody affinity maturation and GC selection were impaired in the presence of the expanded $\mathrm{CD} 11 \mathrm{c}+\mathrm{B}$ cell population. With regard to humans, the increased CD11c+ Tbet+ ABC-like B cell population in patients with SLE that correlates with the frequency of recently activated memory TFH cells (CD4+CXCR5+ICOS+PD-1hi) may represent an important source of the drive towards autoimmunity. ${ }^{14} 38$

Another CD27 IgD- subset sharing some makers of ABC-like B cells, including CD11chi , Tbet+ and lack of CD21, was reported by Jenks et al. ${ }^{14}$ This CXCR5-negative population (called double negative (DN)2) was expanded in SLE, correlated with lupus activity and antiSmith (Sm)/ribonucleoprotein (RNP) autoantibodies. The transcriptional profile found higher expression of IRF4 and lower expression of IFR8 compared to other $\mathrm{B}$ cell subsets, indicating the tendency towards differentiation into PB/PC. Upon TLR7, IL-21 and IL-10 stimulation, DN2 cells produced increased amounts of IgG with a reduced mutation rate than classical switched memory B cells. This indicated that DN2 cells may not originate from the memory B cell compartment. Instead, stimulation of naive B cells with TRL7, IL-21 and IFN $\gamma$ resulted in differentiation into DN2 and $\mathrm{PB} / \mathrm{PC}$.

Another unique B cell subset characterised by CD27Syk++CD38 CD95+ expression and increased basal spleen tyrosine kinase (Syk) phosphorylation was also expanded in patients with SLE but not in RA and pSS, and appeared to be stable over time and did not correlate with disease activity measured by systemic lupus erythematosus disease activity. ${ }^{39}$ Mutated VH gene rearrangements, decreased expression of the ABCB1 transporter and memory like Syk phosphorylation upon BCR stimulation implied that this was an atypical memory population. Comparison of marker expression with other atypical B cell subsets in SLE demonstrated only a partial overlap. Notably, an enhanced frequency of CD27-Syk++ B cells after 48hour stimulation of whole blood cultures with IFN $\gamma$, LPS and TNF suggested that these cells might be expanded in response to inflammatory cytokines. Differentiation into ASCs could be induced by IL-2, IL-10, CpG and anti-BCR 
Table 1 Reported atypical memory B cell subsets reported to be increased in SLE

\begin{tabular}{|c|c|c|c|c|c|c|}
\hline \multirow{2}{*}{$\begin{array}{l}\text { B cell } \\
\text { subset }\end{array}$} & \multirow[b]{2}{*}{ Marker } & \multirow[b]{2}{*}{ Further markers } & \multirow[b]{2}{*}{ Origin } & \multicolumn{2}{|c|}{ B cells } & \multirow[b]{2}{*}{ Function } \\
\hline & & & & HDs & Autoimmunity & \\
\hline $\begin{array}{l}\text { ABC-like } \\
\text { B cells }\end{array}$ & $\begin{array}{l}\mathrm{CD}^{19}{ }^{\text {hi }} \mathrm{CD} 27^{\text {lo }} \\
\mathrm{CD} 21^{\text {lo }} \\
\mathrm{CD} 11 \mathrm{c}^{\text {hi }}\end{array}$ & $\begin{array}{l}\mathrm{CD}^{\text {lo }} \mathrm{CD}^{\mathrm{lo}} 4^{\mathrm{lo}} \\
\mathrm{CD} 38^{\mathrm{lo}} \\
\mathrm{IL}^{\mathrm{lo}} 4 \mathrm{R}^{\mathrm{lo}} \mathrm{CD} 95^{\mathrm{hi}} \\
\mathrm{CD} 86+\mathrm{FCRL} 5+\end{array}$ & $\begin{array}{l}\text { CD27- } \\
\text { memory } \\
\text { B cells }\end{array}$ & $\begin{array}{l}\text { Mean } \\
5 \% * 32\end{array}$ & $\begin{array}{l}\text { CVID (EUROClass; group } \\
\left.\text { SmB } \pm \text { CD21 } 21^{\mathrm{lo}}: \geq 10 \%\right)^{*} \\
\text { SLE ca. } 8-22 \%,{ }^{7} 35 \\
\text { depending on SLEDAI } \\
\text { RA: up to ca. } 17 \%^{34} \\
\text { pSS: median } 8.7 \%^{36}\end{array}$ & $\begin{array}{l}\text { Production of } \\
\text { autoantibodies } \\
\text { Pathogenic role } \\
\text { by abnormal } \\
\text { TFH diff. and GC } \\
\text { selection } \\
38\end{array}$ \\
\hline DN2 & $\begin{array}{l}\text { CD19+ IgD-CD27- } \\
\text { CXCR5-CD24 }\end{array}$ & $\mathrm{See}^{14}$ & Naïve B cells & $\leq 5 \%$ * & SLE: Up to $75 \%$ * & $\begin{array}{l}\text { Production of } \\
\text { autoantibodies } \\
\text { Precursor of } \\
\text { ASCs }\end{array}$ \\
\hline Syk high & $\begin{array}{l}\text { CD19 }{ }^{\text {hi }} C D 20^{\text {hi }} C D 27^{-} \\
\text {Syk }^{\text {hi }} \\
\text { CD38 }^{\text {lo }}\end{array}$ & $\begin{array}{l}\text { pSyk++ } \\
\text { see }^{39}\end{array}$ & $\begin{array}{l}\text { CD27- } \\
\text { memory } \\
\text { B cells }\end{array}$ & $6.4 \%$ & SLE $16.1 \%$ & $\begin{array}{l}\text { Precursor of } \\
\text { ASCs }\end{array}$ \\
\hline
\end{tabular}

*, of peripheral CD19+ B cells.

ABCs, age-/autoimmune-associated B cells; ASCs, antibody-secreting cells; CVID, common variable immunodeficiency; DN, double-negative (CD27- IgD-) B cells; GC, germinal centre; HDs, healthy donors; pSS, primary Sjögren's syndrome; pSyk, phosphorylated spleen tyrosine kinase; SLE, systemic lupus erythematosus; SLEDAI, systemic lupus erythematosus disease activity; Sm, Smith.

stimulation. ${ }^{39}$ The various phenotypes of atypical memory B cells expanded in SLE are shown in table 1.

It is still not clear whether the reported abnormalities in B cell subsets in SLE reflect characteristic findings specifically of this disease or rather are secondary to overactivation of the immune system in patients with SLE. The finding that these cells can be found in other autoimmune as well as infectious diseases and in normal older mice suggests that the common thread might be persistent stimulation of the immune system. However, the finding that DN B cells have been found to be enriched for autoreactive cells against dsDNA, Sm, RNP, and the 9G4 isotype ${ }^{1434}$ suggests that they could contribute to autoimmunity even if arising as part of diffuse B cell activation. Reduced responsiveness to BCR, CD40 or $\mathrm{TLR}^{40}{ }^{41}$ stimulation suggested that they manifested an exhausted anergic or postactivated (APA) phenotype caused by persistent antigen-driven stimulation.

\section{DIMINISHED BCR AND TLR9 SIGNALING IN HUMAN SLE B CELLS}

Engagement of the BCR induces unique organisation of numerous molecules on the surface of B cells leading to intracellular signals that regulate survival, activation, proliferation and differentiation. Together with appropriate second signals by CD40 stimulation and cytokines, the BCR determines B cell fate. Intracellular signalling is defined by stimulatory and inhibitory molecules, including various protein tyrosine kinases (PTKs) and protein tyrosine (serine/threonine) phosphatases (PTPs/PSPs). ${ }^{17-19}$ Antigen binding induces phosphorylation of the BCR-associated Ig $\alpha(\mathrm{CD} 79 \mathrm{a})$ and $\operatorname{Ig} \beta(\mathrm{CD} 79 \mathrm{~b})$ chains leading to downstream Lck/Yes novel tyrosine kinase (Lyn) and Syk phosphorylation. ${ }^{42}$ This activates 1-Phosphatidylinositol-4,5-bisphosphate phosphodiesterase gamma-2 (PLC $\gamma 2)$, Bruton's tyrosine kinase (Btk) as well as protein kinase B (Akt), which results in Ca2+- and Akt-dependent transcription ${ }^{43-45}$ (figure 1). As a negative feedback loop, CD22 becomes phosphorylated and recruits PTP non-receptor type 6 (SHP-1) to the BCR, which dephosphorylates BCR downstream targets. ${ }^{46-48}$ Genome-wide association studies identified polymorphisms of BCR downstream scaffold proteins, PTKs and PTPs, are associated with SLE. ${ }^{49-56}$ These findings suggested that BCR signalling is genetically altered in SLE.

In this context, initial studies reported increased BCR signalling in SLE B cells measured by Ca2+ release and downstream tyrosine phosphorylation related to a lack of negative regulation, such as immunoglobulin gamma Fc receptor II-b (FcgRIIb), phosphatase and tensin homolog or Lyn. ${ }^{20}{ }^{21}{ }^{57-61}$ Further reports provided evidence that the BCR signal in autoimmunity is diminished in certain B cell subsets, with reduced tyrosine phosphorylation, Ca2+ release and recruitment of signalling kinases to lipid rafts upon BCR stimulation. ${ }^{62-65}$ In a recent study, ${ }^{41}$ baseline expression and phosphorylation levels of BCRassociated PTKs, such as Syk, BTK and PLCy2, did not differ from normal in B cells from SLE patients. However, reduced Syk and Btk tyrosine phosphorylation upon BCR stimulation was characteristic of naive and memory B cells from patients with SLE B cells. In contrast, naïve B cells from RA and pSS patients showed similar phosphorylation kinetics as control-naïve B cells. BCR-induced pSyk(Y352) and Btk(Y223) were substantially lower for CD27+ memory B cells and naïve B cells from patients with SLE independent of their surface IgG and IgM expression. ${ }^{64}$ This suggests that this SLE B cell functional phenotype is not related to reduced $\mathrm{Ig}$ 


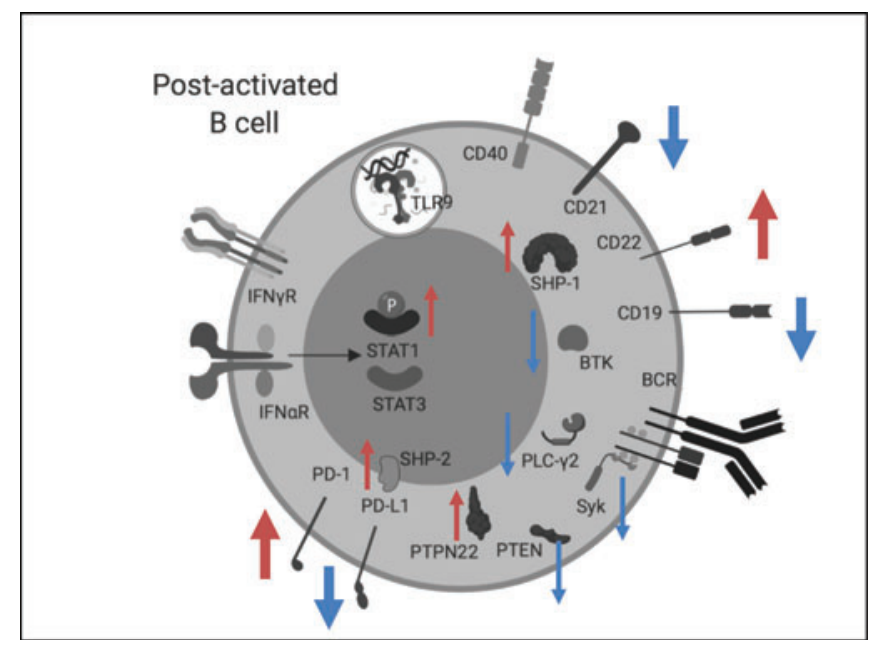

Figure 1 Phenotypic (increased CD22, PD-1 - large red arrows; decreased CD21 and CD19-large blue arrows) and functional characteristics of anergic (postactivated with diminished pSyk, pBTK, pPLC $\gamma 2$ upon BCR activation) B cells expanded for naïve and memory B cells in SLE and memory $B$ cells in RA and primary Sjögren's syndrome. This status is apparently controlled by increased receptor type PTP and generally increased PTP/PSP activity. BCR, B cell receptor; pBTK, phosphorylated Bruton's tyrosine kinase; PLC $\gamma 2$, phosphodiesterase gamma-2; PSP, protein serine/threonine phosphatase; pSyk, phosphorylated spleen tyrosine kinase; PTP, protein tyrosine phosphatase; RA, rheumatoid arthritis.

expression. Moreover, comparable phosphorylation kinetics of $\operatorname{pAkt}\left(\mathrm{S}^{473}\right)$ upon BCR stimulation excluded globally abnormal signalling in lupus B cells.

Mechanistically, repetitive BCR stimulation of B cells from healthy controls, but not with other stimuli such as CpG, resulted into reduced Syk(Y352) phosphorylation upon subsequent BCR stimulation. An earlier study demonstrated that proliferating murine GC B cells lack active BCR signalling which is induced and maintained by increased phosphatase activity and persistent colocalisation of SHP-1 with the BCR after ligation. ${ }^{66}$ Thus, reduced BCR signalling of postactivated functionally anergic B cells likely is induced by repetitive stimulation by self-antigens or immune complexes in the absence of appropriate co-stimulation.

Interestingly, reduced BCR-induced Syk phosphorylation in both CD27- naive and CD27+ memory B cells is related to increased PTP activity and expression by SLE B cells. ${ }^{41}$ This may reflect the more persistent stimulation of B cells in SLE, perhaps reflecting the enhanced activity of $\mathrm{T}$ follicular helper cells (TFH) in SLE $^{67} 68$ and/or exposure to IL-21 that promotes CD11c ${ }^{\text {hi }}$ T-bet+ B cell development. ${ }^{35}$ Even though increased TFH and increased expression of CD40L have been reported in SLE, there may be compartmentalisation of these cells away from sites of B cell activation, permitting persistent engagement of the BCR without appropriate $\mathrm{T}$ cell-derived help.
Increased CD22 and reduced CD21 expression are phenotypic markers of APA B cells ${ }^{39} 6970$ (figure 2). This phenotype is characteristic and a result of the proinflammatory environment, including abundantly available autoantigens in SLE. In this regard, increased PTP as well as PSP activity was a unique finding for SLE B cells. ${ }^{41}$ The data indicate that APA B cells are characterised by increased PTP/PSP activity and explain immediate dephosphorylation of signalling molecules undergoing extrinsic stimulation as an important regulatory mechanism (figure 2). Increased activity of serine/threonine phosphatase, PP2A, has been reported recently to be essential in a preclinical lupus model and was increased in patients with SLE. ${ }^{71}$ Flox/flox PP2A mice had impaired GC formation and TD and TI immune responses, including $\mathrm{PB} / \mathrm{PC}$ formation. Overall, the data indicate that the intracellular PSP/PTP potential is critically involved in mechanisms maintaining SLE cell responsiveness.

Moreover, T cell-independent stimuli employing TLR9 have been reported to enhance B cell activation, in particular when autoantigen/RNP-immune complexes simultaneously engage BCR and TLR9. ${ }^{72}$ Moreover, TLR9 plays a crucial role in breaking tolerance against nuclear antigens and driving B cell activation. ${ }^{73-75}$ In humans, TLR ligands activate memory $\mathrm{B}$ cells, drive in vitro proliferation and differentiation of $\mathrm{B}$ cells into $\mathrm{PB} / \mathrm{PC}^{73} 75$ and is considered to be involved in type I interferon production in autoimmunity. ${ }^{76}$ Hyporesponsiveness to TLR9 in vitro has also been found in SLE B cells ${ }^{41}$ consistent with reduced responses of SLE B cells to pokeweed mitogen ${ }^{77}$; reduced IL-6, IL-10, vascular endothelial growth factor, IL-1ra production and reduced Ki-67 expression ${ }^{78} 79$; reduced frequencies of CD69+CD86+ and TACI+CD25+ B cells. ${ }^{78}$ Interestingly, Syk has been found to be necessary for TLR9 signalling, ${ }^{80} 81$ consistent with the observation that normal B cells showed hyporesponsiveness to CpG when the Syk inhibitor entospletinib was present. ${ }^{41}$ Thus, Syk may connect BCR and TLR activation.

In addition to intrinsic $\mathrm{B}$ cell abnormalities, alteration of the functional status of other cells involved in regulating antibody production may also contribute to the development of autoimmunity. In this regard, abnormal GC reactions in autoimmune tissues, ${ }^{82}$ disturbances of regulatory T cells, ${ }^{2}$ increased $\mathrm{TFH},{ }^{68}$ abnormalities of $\mathrm{CD} 4{ }^{83}$ and $\mathrm{CD} 8+\mathrm{T}$ cells ${ }^{84}{ }^{85}$ with diminished $\mathrm{T}$ cell responses have been reported. As precise understanding of potential abnormalities underlying B cell dysfunction in SLE has critical importance for improved therapeutic outcome, ${ }^{86}$ the operative mechanisms remain to be fully delineated.

\section{ANERGIC B CELLS ARE CHARACTERISED BY INCREASED PTP ACTIVITY CONTROLLED BY CD40 ON THE TRANSCRIPTIONAL LEVEL}

BCR signalling is regulated by a finely tuned balance of PTKs and PTPs, ${ }^{42} 87$ whereas anergic B cells in most patients with SLE are characterised by increased PTP activity, ${ }^{41}{ }^{64}$ and PP2A (PSP) activity ${ }^{71}$ (figure 2). Of 


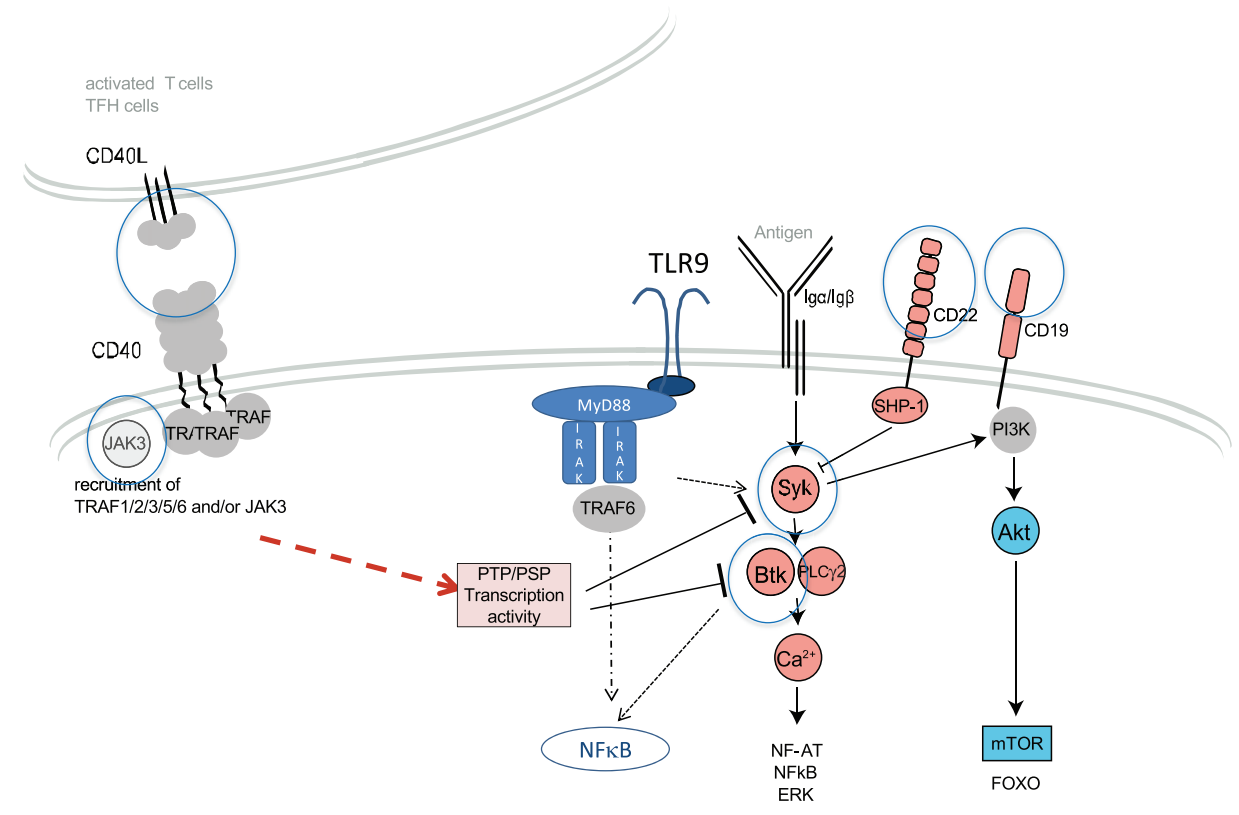

Figure 2 The BCR complex signalling pathway and TLR9 signalling share PTKs, that is, Syk and BTK. CD40 stimulation is able to decrease intracellular PTP and PSP activity in B cells together with receptor-type phosphatases controlling anergic B cells that are expanded in SLE. BCR, B cell receptor; BTK, Bruton's tyrosine kinase; PSP, protein serine/threonine phosphatase; PTKs, protein tyrosine kinases; PTP, protein tyrosine phosphatase; Syk, spleen tyrosine kinase; TLR, toll-like receptor.

note, PTP and PSP activities of CD3+ T cells are not increased. Increased PTP activity is also evidenced by elevated co-localisation of SHP-1 (PTPN6) with CD22 on the B cell surface. The degree of co-localisation constitutively present in SLE B cells appeared at a maximum, cannot be further increased upon CD22 engagement and suggest that a functionally active PTP complex of SHP-1 is substantially increased in SLE B cells.

\section{CO-STIMULATION OF CD40 EFFECTIVELY IMPROVES B CELL ANERGY IN SLE}

Gene expression analysis identified increased PTPN2, PTPN11, PTPN22, PTPRC and PTPRO in SLE CD20+ B cells. Of particular note, CD40L/IL-4 stimulation resulted in reduced transcription of PTPN2, PTPN22 and almost all receptor-related (R)PTP (except PTPRB) compared to their overexpression before stimulation. The available data of PSPs are limited, and no clear overexpression or downregulation upon CD40 stimulation was observed. However, the PSP PP2A has previously been reported to be overexpressed and functionally relevant in SLE. ${ }^{71}$

Thus, T cell help by CD40/IL-4R engagement alters the expression of NRPTPs, such as PTPN22, and different RPTPs and supports the crucial role of $\mathrm{T}$ cell costimulation in defining B cell dysfunction in SLE. Some reports also indicate a role of reduced BCR signalling in the development and progression of autoimmunity. ${ }^{88} 89$ Interestingly, inhibition of PTPN22 could reset central B cell tolerance in Non-obese diabetic (NOD) scid gamma chain knock out mice which were engrafted with human haematopoietic stem cells carrying the gain of function mutation of PTPN22. ${ }^{89}$ Whereas this study indicates that a normalised BCR signal could restore immune tolerance, the strong role of CD40 activation in PTPN22 risk gene carriers is also consistent with the idea that this pathway is critical for censoring the overly active immune system in autoimmunity. ${ }^{90}$ Whether PTPN22 variant increases or decreases BCR signalling is a matter of debate. Mice expressing this mutation displayed enhanced BCR and CD40 responses. ${ }^{91}$ CD40 seems to be a critical context-dependent co-stimulatory molecule regulating both the full activation of BCR-stimulated $\mathrm{B}$ cells as well as their subsequent censoring.

CD40 co-stimulation, in contrast to CpG or cytokine stimulation alone (IL-4, B cell activating factor from the TNF family (BAFF), IL-6, IL-21), improved BCR responsiveness, including Syk(Y352) phosphorylation in CD27-naïve and CD27+ memory B cells from patients with SLE. This data suggests improved BCR responsiveness by CD40L co-stimulation in memory $\mathrm{B}$ cells and naive SLE cells. Before these results were obtained, it has been reported that Th2 signals restore BCR signalling in a small population of anergic IgM-IgD+-naïve $\mathrm{B}$ cells present in blood of healthy donors and patients with SLE. ${ }^{92}$ IL-4 or IL-21 alone led to modest effects on the pSyk(Y352) response to BCR engagement. IL-4 in combination with $\mathrm{CD} 40 \mathrm{~L}$, however, led to higher responses than with $\mathrm{CD} 40 \mathrm{~L}$ alone.

Co-stimulation of CD40 also increased restored TLR9 induced proliferation of SLE B cells. As already 
reported ${ }^{93}$ the induction of $\mathrm{CD} 27+\mathrm{CD} 38+\mathrm{PB} / \mathrm{PC}$ was lower compared to TLR9 or TLR9/BCR stimulation. Of note, however, $\mathrm{CD} 40 \mathrm{~L}$ stimulation blocked $\mathrm{CpG}$ induced in vitro B cell differentiation to $\mathrm{PB} / \mathrm{PC}$. Therefore, CD40 engagement provided the co-stimulation signal that allowed SLE B cells to proliferate when co-stimulated through TLR9 but did not promote differentiation into CD27+CD38+ PB/PC.

In summary, CD40 engagement on SLE B cells improves largely their hyporesponsiveness to BCR and TLR9 agonists and appears to be a checkpoint molecule controlling anergic $\mathrm{B}$ cell fate. This conclusion is further supported by the observation that in addition to repeated BCR engagement, continuous signalling via SHP-1 is required to maintain anergy ${ }^{94}{ }^{95}$ As the APA B cells appear to be functionally reverted by CD40 engagement, there is the likelihood that the anergic state derives from signalling through the BCR in vivo without appropriate $\mathrm{T}$ cell-derived co-stimulation. Moreover, since APA B cells are enriched in reactivity to autoantigens, functional anergy may be an important brake on the development of autoimmunity. Bystander help through CD40 may provide the signal to overcome anergy and permit the emergence of autoimmunity. Thus, the regulation of proper T-B interaction plays an essential role both in assuring a response to exogenous antigens but also in controlling autoimmunity and the production of autoantibodies in SLE.

These observations have important consequences. First, most mature B cells in SLE are APA B cells regardless of the stage of differentiation. This functional state does not appear to be restricted to ABC-like B cells. Second, the presence of APA B cells does not appear to be secondary to disease activity as this functional phenotype is found in patients with both active and inactive disease. Moreover, increased APA B cells can also be found in patients with other autoimmune disease, including RA and pSS, and, therefore, it is not specific for SLE. ${ }^{41}$ Third, anergy is characterised by upregulation of inhibitory molecules as well as protein tyrosine phosphatases and can be reversed by inhibition of the dephosphorylation of signalling molecules. Finally, anergy appears to be specific for signalling through the BCR, as the cells can partially respond to TLR engagement and can be functionally restored by signalling through CD40. These findings have numerous implications. First, ongoing activation of all B cell subsets, presumably owing to chronic autoantigen exposure, seems to be a characteristic of SLE and other autoimmune diseases. Second, in so far as APA B cells contain subsets with reactivity to autoantigens, they might be rescued by bystander help provided by activated $\mathrm{T}$ cells expressing CD40L and be induced to differentiate into autoantibodyproducing $\mathrm{PB} / \mathrm{PC}$.

It is notable that anergy is not restricted to B cells in SLE and might contribute at least in part to the increased infectious risks in this condition. Impaired cytotoxic function and exhaustion of CD8+ T cells, a characteristic of viral infections, has been reported. ${ }^{84} 8596$ Moreover, SLE T cells display abnormal T cell signalling. ${ }^{83}{ }^{97}$ In addition, exhausted CD4+ T cells with dysbalanced IL-17/IL-2 production have been reported. ${ }^{83}$ The full impact of a broadly anergic adaptive immune system in SLE has not been fully delineated.

\section{THERAPEUTIC IMPLICATIONS}

The state of B cell anergy with its increased phosphatase activity provides translational guidance for innovative therapies as well as possible explanations for some failed SLE trials. The generalised increase in APA B cells suggests that these populations are stimulated through the BCR in vivo. However, little is known about this process and targeting it might require prolonged time for an effect to become manifest. There is, however, evidence that the APA phenotype can be reversed in vivo by certain therapeutic interventions. In this regard, reduced cytokine production by RA B cells appeared to be reversible upon IL-6R blockade ${ }^{98}$ and anergic memory B cells in RA were decreased. ${ }^{41}$ However, whether other approaches to block B cell activation, such as inhibition of BTK, will be effective in altering the APA B cell phenotype is uncertain. Moreover, whether such inhibition will render $\mathrm{B}$ cells responsive to subsequent stimulation is unclear. In the context of recent approaches to inhibit downstream BCR signalling, studies using inhibitors of BTK in SLE (fenebrutinib) ${ }^{99}$ as well as BTK in RA (fenebrutinib) ${ }^{100}$ and Syk (fostamatinib) did not differentiate substantially from placebo. ${ }^{101}$ It is possible that these agents are not effective at targeting APA B cells or inhibit the in vivo generation of APA B cells leaving the patient able to fully respond to expressed autoantigens. Of particular note, the Syk inhibitor (fostamatinib) has been approved for autoimmune thrombocytopenia ${ }^{102}$ and the Btk inhibitor evobrutinib is in multiple sclerosis phase III trials after successful initial studies. ${ }^{103}$ The latter two organ-specific autoimmune diseases may differ from SLE and RA regarding the role of APA B cells.

The failure of epratuzumab (anti-CD22) ${ }^{104}$ can also be considered in the context of the prevalence of APA B cells in SLE. It was thought that CD22 engagement would impose negative regulation of $\mathrm{BCR}$ signalling ${ }^{47}$ by increased SHP-1 activity. This could be convincingly shown for normal B cells ${ }^{47105}$ but since CD22 is fully engaged in SLE APA B cells and phosphatases are upregulated, ${ }^{41}$ the inability of epratuzumab to downregulate APA B cell function is perhaps understandable.

Experiences with belimumab, an anti-BAFF/B-lymphocyte stimulator (BLyS) antibody, showed an impact on the differentiation of mature naive and autoreactive B cells. ${ }^{106}$ An open study of 23 patients with SLE evaluated alterations of leucocyte subsets under belimumab using mass cytometry (CyTOF) ${ }^{107}$ A rapid decrease of naïve B cells, a gradual decrease of DN B cells, but no substantial $\mathrm{T}$ cell changes were noted, which largely confirmed previous studies. Intriguing new insights were reported for ABC-like B cells expressing $\mathrm{BAFF} / \mathrm{BLyS}$ receptors ${ }^{16}{ }^{35}$ under this treatment. CD11c+CD21- ABC-like B cells together with IgA+ 
memory B cells decreased early with further declines over the observation period of 24 months. The early decline of both subsets was found especially in early responders with no clear dynamics in non-responders and delayed responders. Further studies are warranted to delineate how belimumab might impact on ABC-like B cells and perhaps APA $\mathrm{B}$ cells as well, and whether $\mathrm{CD} 11 \mathrm{C}+\mathrm{CD} 21-\mathrm{ABC}$-like B cells and IgA+ memory B cells are interlinked.

The ability of CD40 engagement to restore functional activity to APA B cells suggests that blocking this pathway may be an effective means to limit rescues of APA B cell function in SLE. Initial proof of concept was obtained by a CD154 monoclonal in lupus nephritis, ${ }^{108}$ but the impact of this intervention on $\mathrm{PB} / \mathrm{PC}$ was the focus of investigation. ${ }^{109}$ The induction of thromboembolic events prevented further studies at that time, but a second-generation PEGylated anti-CD154 antibody is currently in clinical development for SLE. ${ }^{110}{ }^{111}$ Antibodies blocking CD40 are also in clinical development. Despite blocking CD40 or the ligand CD154 on the extracellular or receptor level, intracellular and selective targeting of CD40 downstream pathways holds additional therapeutic promise.

\section{CONCLUSION}

The full impact of a broadly anergic adaptive immune system and the recent identification of an APA B cell phenotype in SLE has not be fully delineated. Innovative treatment approaches should take into consideration that adaptive immunity is globally hyporesponsive. Identification of key pathways able to overcome or overrule this status can explain the expansion of autoreactive B cell clones where the cognate autoantigen is not an essential driver of the autoimmune process, but rather bystander help provided by CD40/CD154 interactions can rescue APA B cells leading to the generation of autoantibodyproducing $\mathrm{PB} / \mathrm{PC}$. While the anergic adaptive immunity also suggests impaired protective immunity, further studies of regulatory principles to maintain or control APA $B$ cells hold promise for more effective and safe treatments in SLE.

Contributors All authors developed the concept of the review, have been involved in drafting, revising and critically reviewing the manuscript and its revision.

Funding The DRFZ is funded by the institute of the Leibniz-Gesellschaft. The review has been funded in part by DFG grant Do491/11-1, 10-2, 7-4 and a Transregio 130 project to TD.

Competing interests None declared.

Patient consent for publication Not required.

Provenance and peer review Commissioned; externally peer reviewed.

Open access This is an open access article distributed in accordance with the Creative Commons Attribution Non Commercial (CC BY-NC 4.0) license, which permits others to distribute, remix, adapt, build upon this work non-commercially, and license their derivative works on different terms, provided the original work is properly cited, appropriate credit is given, any changes made indicated, and the use is non-commercial. See: http://creativecommons.org/licenses/by-nc/4.0/.

\section{ORCID iDs}

Thomas Dörner http://orcid.org/0000-0002-6478-7725

\section{REFERENCES}

1 Dorner T, Lipsky PE. B cells: depletion or functional modulation in rheumatic diseases. Curr Opin Rheumatol 2014;26:228-36.

2 Wahren-Herlenius M, Dorner T. Immunopathogenic mechanisms of systemic autoimmune disease. Lancet 2013;382:819-31.

3 Shlomchik MJ, Madaio MP. The role of antibodies and B cells in the pathogenesis of lupus nephritis. Springer Semin Immunopathol 2003;24:363-75.

4 Liu Z, Davidson A. IFNalpha inducible models of murine SLE. Front Immunol 2013;4:306.

5 Jacobi AM, Odendahl M, Reiter K, et al. Correlation between circulating CD27high plasma cells and disease activity in patients with systemic lupus erythematosus. Arthritis Rheum 2003;48:1332-42.

6 Jacobi AM, Mei H, Hoyer BF, et al. HLA-DRhigh/CD27high plasmablasts indicate active disease in patients with systemic lupus erythematosus. Ann Rheum Dis 2010;69:305-8.

7 Odendahl M, Keitzer R, Wahn U, et al. Perturbations of peripheral B lymphocyte homoeostasis in children with systemic lupus erythematosus. Ann Rheum Dis 2003;62:851-8.

8 Odendahl M, Jacobi A, Hansen A, et al. Disturbed peripheral B lymphocyte homeostasis in systemic lupus erythematosus. $\mathrm{J}$ Immunol 2000;165:5970-9.

9 Jacobi AM, Reiter K, Mackay M, et al. Activated memory B cell subsets correlate with disease activity in systemic lupus erythematosus: delineation by expression of CD27, IgD, and CD95. Arthritis Rheum 2008;58:1762-73.

10 Wei C, Anolik J, Cappione A, et al. A new population of cells lacking expression of CD27 represents a notable component of the B cell memory compartment in systemic lupus erythematosus. $J$ Immunol 2007;178:6624-33.

11 Yeo L, Lom H, Juarez M, et al. Expression of FcRL4 defines a pro-inflammatory, RANKL-producing B cell subset in rheumatoid arthritis. Ann Rheum Dis 2015;74:928-35

12 Hansen A, Odendahl M, Reiter K, et al. Diminished peripheral blood memory B cells and accumulation of memory B cells in the salivary glands of patients with Sjogren's syndrome. Arthritis Rheum 2002;46:2160-71.

13 Bohnhorst JO, Bjorgan MB, Thoen JE, et al. Bm1-Bm5 classification of peripheral blood $\mathrm{B}$ cells reveals circulating germinal center founder cells in healthy individuals and disturbance in the B cell subpopulations in patients with primary Sjogren's syndrome. $J$ Immunol 2001;167:3610-8.

14 Jenks SA, Cashman KS, Zumaquero E, et al. Distinct effector B cells induced by unregulated toll-like receptor 7 contribute to pathogenic responses in systemic lupus erythematosus. Immunity 2018;49:72539 e6.

15 Tipton CM, Fucile CF, Darce J, et al. Diversity, cellular origin and autoreactivity of antibody-secreting cell population expansions in acute systemic lupus erythematosus. Nat Immunol 2015;16:755-65.

16 Rubtsova K, Rubtsov AV, Cancro MP, et al. Age-associated B cells: a T-bet-dependent effector with roles in protective and pathogenic immunity. J Immunol 2015;195:1933-7.

17 Casola S, Otipoby KL, Alimzhanov M, et al. B cell receptor signal strength determines B cell fate. Nat Immunol 2004;5:317-27.

18 Paus D, Phan TG, Chan TD, et al. Antigen recognition strength regulates the choice between extrafollicular plasma cell and germinal center B cell differentiation. J Exp Med 2006;203:1081-91.

19 Shinnakasu R, Kurosaki T. Regulation of memory B and plasma cell differentiation. Curr Opin Immunol 2017;45:126-31.

20 Liossis SN, Kovacs B, Dennis G, et al. B cells from patients with systemic lupus erythematosus display abnormal antigen receptor-mediated early signal transduction events. J Clin Invest 1996;98:2549-57.

21 Wu XN, Ye YX, Niu JW, et al. Defective PTEN regulation contributes to B cell hyperresponsiveness in systemic lupus erythematosus. Sci Transl Med 2014;6:246ra99.

22 Galligan CL, Siebert JC, Siminovitch KA, et al. Multiparameter phospho-flow analysis of lymphocytes in early rheumatoid arthritis: implications for diagnosis and monitoring drug therapy. PLoS One 2009;4:e6703.

23 Zikherman J, Weiss A. Antigen receptor signaling in the rheumatic diseases. Arthritis Res Ther 2009;11:202.

24 Dorner T, Jacobi AM, Lee J, et al. Abnormalities of B cell subsets in patients with systemic lupus erythematosus. J Immunol Methods 2011;363:187-97.

25 Banchereau R, Hong S, Cantarel B, et al. Personalized immunomonitoring uncovers molecular networks that stratify lupus patients. Cell 2016;165:551-65.

26 Mei HE, Hahne S, Redlin A, et al. Plasmablasts with a mucosal phenotype contribute to plasmacytosis in systemic lupus erythematosus. Arthritis Rheumatol 2017;69:2018-28. 
27 Cossarizza A, Chang HD, Radbruch A, et al. Guidelines for the use of flow cytometry and cell sorting in immunological studies (second edition). Eur J Immunol 2019;49:1457-973.

28 Sanz I, Wei C, Jenks SA, et al. Challenges and opportunities for consistent classification of human B cell and plasma cell populations. Front Immunol 2019;10:2458.

29 Mahmood Z, Muhammad K, Schmalzing M, et al. CD27-lgD- memory $B$ cells are modulated by in vivo interleukin-6 receptor (IL-6R) blockade in rheumatoid arthritis. Arthritis Res Ther 2015;17:61.

30 Rubin SJS, Bai L, Haileselassie Y, et al. Mass cytometry reveals systemic and local immune signatures that distinguish inflammatory bowel diseases. Nat Commun 2019;10:2686.

31 Cancro MP. Age-associated B cells. Annu Rev Immunol 2020;38:315-40.

32 Thorarinsdottir K, Camponeschi A, Gjertsson I, et al. CD21 -/low $B$ cells: a snapshot of a unique b cell subset in health and disease. Scand J Immunol 2015;82:254-61.

$33 \mathrm{Li} \mathrm{H}$, Borrego F, Nagata S, et al. Fc receptor-like 5 expression distinguishes two distinct subsets of human circulating tissue-like memory B cells. J Immunol 2016;196:4064-74.

34 Isnardi I, Ng YS, Menard L, et al. Complement receptor 2/CD21human naive $B$ cells contain mostly autoreactive unresponsive clones. Blood 2010;115:5026-36.

35 Wang S, Wang J, Kumar V, et al. IL-21 drives expansion and plasma cell differentiation of autoreactive CD11c(hi)T-bet(+) B cells in SLE. Nat Commun 2018;9:1758.

36 Saadoun D, Terrier B, Bannock J, et al. Expansion of autoreactive unresponsive CD21-/low B cells in Sjogren's syndrome-associated lymphoproliferation. Arthritis Rheum 2013;65:1085-96.

37 Weiss GE, Crompton PD, Li S, et al. Atypical memory B cells are greatly expanded in individuals living in a malaria-endemic area. $\mathrm{J}$ Immunol 2009;183:2176-82.

38 Zhang W, Zhang H, Liu S, et al. Excessive CD11c(+)Tbet(+) B cells promote aberrant TFH differentiation and affinity-based germinal center selection in lupus. Proc Natl Acad Sci U S A 2019;116:18550-60.

39 Fleischer SJ, Giesecke C, Mei HE, et al. Increased frequency of a unique spleen tyrosine kinase bright memory B cell population in systemic lupus erythematosus. Arthritis Rheumatol 2014;66:3424-35.

40 Thorarinsdottir K, Camponeschi A, Cavallini N, et al. CD21(-/low) $B$ cells in human blood are memory cells. Clin Exp Immunol 2016;185:252-62.

41 Weissenberg SY, Szelinski F, Schrezenmeier E, et al. Identification and characterization of post-activated B cells in systemic autoimmune diseases. Front Immunol 2019;10:2136.

42 Rolli V, Gallwitz M, Wossning T, et al. Amplification of B cell antigen receptor signaling by a Syk/ITAM positive feedback loop. Mol Cell 2002;10:1057-69.

43 Toapanta FR, Bernal PJ, Sztein MB. Diverse phosphorylation patterns of $B$ cell receptor-associated signaling in naive and memory human B cells revealed by phosphoflow, a powerful technique to study signaling at the single cell level. Front Cell Infect Microbiol 2012;2:128.

44 Scharenberg AM, Humphries LA, Rawlings DJ. Calcium signalling and cell-fate choice in B cells. Nat Rev Immunol 2007;7:778-89.

45 Pogue SL, Kurosaki T, Bolen J, et al. B cell antigen receptor-induced activation of Akt promotes B cell survival and is dependent on Syk kinase. J Immunol 2000;165:1300-6.

46 Muller J, Obermeier I, Wohner M, et al. CD22 ligand-binding and signaling domains reciprocally regulate $\mathrm{B}-$ cell $\mathrm{Ca} 2+$ signaling. Proc Natl Acad Sci U S A 2013;110:12402-7

47 Sieger N, Fleischer SJ, Mei HE, et al. CD22 ligation inhibits downstream $\mathrm{B}$ cell receptor signaling and $\mathrm{Ca} 2+$ flux upon activation. Arthritis Rheum 2013:65:770-9.

48 Poe JC, Fujimoto Y, Hasegawa M, et al. CD22 regulates $B$ lymphocyte function in vivo through both ligand-dependent and ligand-independent mechanisms. Nat Immunol 2004;5:1078-87.

49 Vaughn SE, Kottyan LC, Munroe ME, et al. Genetic susceptibility to lupus: the biological basis of genetic risk found in B cell signaling pathways. J Leukoc Biol 2012;92:577-91.

50 Manjarrez-Orduno N, Marasco E, Chung SA, et al. CSK regulatory polymorphism is associated with systemic lupus erythematosus and influences B-cell signaling and activation. Nat Genet 2012;44:1227-30.

51 Jarvinen TM, Hellquist A, Zucchelli M, et al. Replication of GWASidentified systemic lupus erythematosus susceptibility genes affirms B-cell receptor pathway signalling and strengthens the role of IRF5 in disease susceptibility in a Northern European population. Rheumatology 2012;51:87-92
52 Lu R, Vidal GS, Kelly JA, et al. Genetic associations of LYN with systemic lupus erythematosus. Genes Immun 2009;10:397-403.

53 Kozyrev SV, Abelson AK, Wojcik J, et al. Functional variants in the B -cell gene BANK1 are associated with systemic lupus erythematosus. Nat Genet 2008;40:211-6.

54 Orozco G, Eyre S, Hinks A, et al. Study of the common genetic background for rheumatoid arthritis and systemic lupus erythematosus. Ann Rheum Dis 2011;70:463-8.

55 Sun F, Xu J, Wu Z, et al. Polymorphisms in the FAM167A-BLK, but not BANK1, are associated with primary Sjogren's syndrome in a Han Chinese population. Clin Exp Rheumatol 2013;31:70410.

56 Burbelo PD, Ambatipudi K, Alevizos I. Genome-wide association studies in Sjogren's syndrome: what do the genes tell us about disease pathogenesis? Autoimmun Rev 2014;13:756-61.

57 Liossis SN, Solomou EE, Dimopoulos MA, et al. B-cell kinase Lyn deficiency in patients with systemic lupus erythematosus. J Investig Med 2001;49:157-65.

58 Su K, Yang H, Li X, et al. Expression profile of FcgammaRIlb on leukocytes and its dysregulation in systemic lupus erythematosus. J Immunol 2007; 178: 3272-80.

59 Mackay M, Stanevsky A, Wang T, et al. Selective dysregulation of the FcgammallB receptor on memory B cells in SLE. J Exp Med 2006;203:2157-64.

60 Enyedy EJ, Mitchell JP, Nambiar MP, et al. Defective FcgammaRIIb1 signaling contributes to enhanced calcium response in B cells from patients with systemic lupus erythematosus. Clin Immunol 2001;101:130-5

61 Anzelon AN, Wu H, Rickert RC. Pten inactivation alters peripheral B lymphocyte fate and reconstitutes CD19 function. Nat Immunol 2003;4:287-94.

62 Dam EM, Habib T, Chen J, et al. The BANK1 SLE-risk variants are associated with alterations in peripheral B cell signaling and development in humans. Clin Immunol 2016;173:171-80.

63 Arechiga AF, Habib T, He Y, et al. Cutting edge: the PTPN22 allelic variant associated with autoimmunity impairs $B$ cell signaling. $\mathrm{J}$ Immunol 2009;182:3343-7.

64 Fleischer SJ, Daridon C, Fleischer V, et al. Enhanced tyrosine phosphatase activity underlies dysregulated $B$ cell receptor signaling and promotes survival of human lupus B cells. Arthritis Rheumatol 2016;68:1210-21.

65 Vasquez A, Baena A, Gonzalez LA, et al. Altered recruitment of Lyn, Syk and ZAP-70 into lipid rafts of activated B cells in systemic lupus erythematosus. Cell Signal 2019;58:9-19.

66 Khalil AM, Cambier JC, Shlomchik MJ. B cell receptor signal transduction in the GC is short-circuited by high phosphatase activity. Science 2012;336:1178-81.

67 Gensous N, Charrier M, Duluc D, et al. T follicular helper cells in autoimmune disorders. Front Immunol 2018;9:1637.

68 Choi JY, Ho JH, Pasoto SG, et al. Circulating follicular helper-like T cells in systemic lupus erythematosus: association with disease activity. Arthritis Rheumatol 2015;67:988-99.

69 Keller B, Stumpf I, Strohmeier V, et al. High SYK expression drives constitutive activation of CD21 low B cells. J Immunol 2017;198:4285-92.

70 Wehr C, Kivioja T, Schmitt C, et al. The EUROclass trial: defining subgroups in common variable immunodeficiency. Blood 2008;111:77-85.

71 Meidan E, Li H, Pan W, et al. Serine/threonine phosphatase PP2A is essential for optimal B cell function. JCI Insight 2020;5.

72 Bengtsson AA, Ronnblom L. Role of interferons in SLE. Best Pract Res Clin Rheumatol 2017;31:415-28.

73 Bernasconi NL, Onai N, Lanzavecchia A. A role for Toll-like receptors in acquired immunity: up-regulation of TLR9 by BCR triggering in naive $B$ cells and constitutive expression in memory $B$ cells. Blood 2003;101:4500-4.

74 Busconi L, Bauer JW, Tumang JR, et al. Functional outcome of B cell activation by chromatin immune complex engagement of the $B$ cell receptor and TLR9. J Immunol 2007;179:7397-405.

75 Viglianti GA, Lau CM, Hanley TM, et al. Activation of autoreactive B cells by CpG dsDNA. Immunity 2003; 19:837-47.

76 Jacquemin C, Schmitt N, Contin-Bordes C, et al. OX40 ligand contributes to human lupus pathogenesis by promoting $t$ follicular helper response. Immunity 2015:42:1159-70.

77 Ginsburg WW, Finkelman FD, Lipsky PE. Circulating and pokeweed mitogen-induced immunoglobulin-secreting cells in systemic lupus erythematosus. Clin Exp Immunol 1979;35:76-88.

78 Gies V, Schickel JN, Jung S, et al. Impaired TLR9 responses in B cells from patients with systemic lupus erythematosus. JCI Insight 2018;3.

79 Sieber J, Daridon C, Fleischer SJ, et al. Active systemic lupus erythematosus is associated with a reduced cytokine production by 
B cells in response to TLR9 stimulation. Arthritis Res Ther 2014;16:477.

80 Kremlitzka M, Macsik-Valent B, Erdei A. Syk is indispensable for CpG-induced activation and differentiation of human B cells. Cell Mol Life Sci 2015;72:2223-36.

81 Iwata S, Yamaoka K, Niiro H, et al. Amplification of Toll-like receptormediated signaling through spleen tyrosine kinase in human B-cell activation. J Allergy Clin Immunol 2012;129:1594-601 e2.

82 Tipton CM, Hom JR, Fucile CF, et al. Understanding B-cell activation and autoantibody repertoire selection in systemic lupus erythematosus: a B-cell immunomics approach. Immunol Rev 2018;284:120-31.

83 Katsuyama T, Tsokos GC, Moulton VR. Aberrant T cell signaling and subsets in systemic lupus erythematosus. Front Immunol 2018;9:1088.

84 McKinney EF, Lee JC, Jayne DR, et al. T-cell exhaustion, co-stimulation and clinical outcome in autoimmunity and infection. Nature 2015;523:612-6.

85 Comte D, Karampetsou MP, Yoshida N, et al. Signaling lymphocytic activation molecule family member 7 engagement restores defective effector CD8+ T cell function in systemic lupus erythematosus. Arthritis Rheumatol 2017;69:1035-44.

86 Schrezenmeier E, Weissenberg SY, Stefanski AL, et al. Postactivated $B$ cells in systemic lupus erythematosus: update on translational aspects and therapeutic considerations. Curr Opin Rheumatol 2019;31:175-84.

87 Alsadeq A, Hobeika E, Medgyesi D, et al. The role of the Syk/Shp-1 kinase-phosphatase equilibrium in B cell development and signaling. J Immunol 2014;193:268-76.

88 Konigsberger S, Prodohl J, Stegner D, et al. Altered BCR signalling quality predisposes to autoimmune disease and a pre-diabetic state. Embo J 2012;31:3363-74.

89 Schickel JN, Kuhny M, Baldo A, et al. PTPN22 inhibition resets defective human central B cell tolerance. Sci Immunol 2016;1: aaf7153-aaf7153-

90 Menard L, Saadoun D, Isnardi I, et al. The PTPN22 allele encoding an R620W variant interferes with the removal of developing autoreactive B cells in humans. J Clin Invest 2011;121:3635-44.

91 Metzler G, Dai X, Thouvenel CD, et al. The autoimmune risk variant PTPN22 C1858T alters B cell tolerance at discrete checkpoints and differentially shapes the naive repertoire. $J$ Immunol 2017;199:2249-60.

92 Szodoray P, Stanford SM, Molberg O, et al. T-helper signals restore $\mathrm{B}$-cell receptor signaling in autoreactive anergic $\mathrm{B}$ cells by upregulating CD45 phosphatase activity. J Allergy Clin Immunol 2016;138:839-51 e8.

93 Marasco E, Farroni C, Cascioli S, et al. B-cell activation with CD40L or $\mathrm{CpG}$ measures the function of B-cell subsets and identifies specific defects in immunodeficient patients. Eur J Immunol 2017;47:131-43.

94 Getahun A, Beavers NA, Larson SR, et al. Continuous inhibitory signaling by both SHP-1 and SHIP-1 pathways is required to maintain unresponsiveness of anergic B cells. J Exp Med 2016;213:751-69.

95 Franks SE, Cambier JC. Putting on the brakes: regulatory kinases and phosphatases maintaining B cell anergy. Front Immunol 2018;9:665

96 Kis-Toth K, Comte D, Karampetsou MP, et al. Selective loss of signaling lymphocytic activation molecule family member 4-positive CD8+ $\mathrm{T}$ cells contributes to the decreased cytotoxic cell activity in systemic lupus erythematosus. Arthritis Rheumatol 2016;68:164-73.

97 Sharabi A, Kasper IR, Tsokos GC. The serine/threonine protein phosphatase $2 \mathrm{~A}$ controls autoimmunity. Clin Immunol 2018;186:38-42.

98 Fleischer S, Ries S, Shen P, et al. Anti-interleukin-6 signalling therapy rebalances the disrupted cytokine production of $B$ cells from patients with active rheumatoid arthritis. Eur J Immunol 2018;48:194-203.

99 Isenberg D, et al, ACR 2019, Arthritis Rheumato 2019;7: suppl 10.

100 Cohen S et al, EULAR 2019; OP0025.

101 Weinblatt ME, Genovese MC, Ho M, et al. Effects of fostamatinib, an oral spleen tyrosine kinase inhibitor, in rheumatoid arthritis patients with an inadequate response to methotrexate: results from a phase III, multicenter, randomized, double-blind, placebo-controlled, parallel-group study. Arthritis Rheumatol 2014;66:3255-64.

102 Bussel J, Arnold DM, Grossbard E, et al. Fostamatinib for the treatment of adult persistent and chronic immune thrombocytopenia: results of two phase 3 , randomized, placebo-controlled trials. Am $J$ Hematol 2018;93:921-30.

103 Montalban X, Arnold DL, Weber MS, et al. Placebo-controlled trial of an oral BTK inhibitor in multiple sclerosis. N Engl J Med 2019;380:2406-17.

104 Clowse ME, Wallace DJ, Furie RA, et al. Efficacy and safety of epratuzumab in moderately to severely active systemic lupus erythematosus: results from two phase III randomized, double-blind, placebo-controlled trials. Arthritis Rheumatol 2017:69:362-75.

105 Lumb S, Fleischer SJ, Wiedemann A, et al. Engagement of CD22 on $B$ cells with the monoclonal antibody epratuzumab stimulates the phosphorylation of upstream inhibitory signals of the B cell receptor. J Cell Commun Signal 2016;10:143-51.

106 Huang W, Quach TD, Dascalu C, et al. Belimumab promotes negative selection of activated autoreactive $B$ cells in systemic lupus erythematosus patients. JCl Insight 2018;3.

107 Ramskold D, Parodis I, Lakshmikanth T, et al. B cell alterations during BAFF inhibition with belimumab in SLE. EBioMedicine 2019;40:517-27.

108 Boumpas DT, Furie R, Manzi S, et al. A short course of BG9588 (anti-CD40 ligand antibody) improves serologic activity and decreases hematuria in patients with proliferative lupus glomerulonephritis. Arthritis Rheum 2003;48:719-27.

109 Grammer AC, Slota R, Fischer R, et al. Abnormal germinal center reactions in systemic lupus erythematosus demonstrated by blockade of CD154-CD40 interactions. J Clin Invest 2003;112:1506-20.

110 Chamberlain C, Colman PJ, Ranger AM, et al. Repeated administration of dapirolizumab pegol in a randomised phase I study is well tolerated and accompanied by improvements in several composite measures of systemic lupus erythematosus disease activity and changes in whole blood transcriptomic profiles. Ann Rheum Dis 2017;76:1837-44.

111 Furie et al, EULAR 2019, FRI0195. Ann Rheum Dis 78 (Suppl 2):775.2-776 